\title{
Screening CT angiography in patients with suspected fibromuscular dysplasia: improved patient care with single-session skull vertex to pelvis coverage
}

\author{
Mostafa Abozeed $^{1,2}$, Michael A. Bolen ${ }^{1}$ \\ ${ }^{1}$ Imaging Institute, Cleveland Clinic, Cleveland, OH, USA; ${ }^{2}$ Radiology Department, Al-Azhar University, Cairo, Egypt \\ Contributions: (I) Conception and design: All authors; (II) Administrative support: None; (III) Provision of study materials or patients: None; (IV) \\ Collection and assembly of data: M Abozeed; (V) Data analysis and interpretation: All authors; (VI) Manuscript writing: All authors; (VII) Final \\ approval of manuscript: All authors. \\ Correspondence to: Michael A. Bolen, MD. Imaging Institute, Cleveland Clinic, Cleveland, OH 44195, USA. Email: bolenm@ccf.org.
}

Background: Imaging plays a key role in the workup of patients with clinically suspected fibromuscular
dysplasia (FMD), and research has highlighted the potential of computed tomography angiography (CTA) in
screening for thoracic, abdominal, and pelvic arterial abnormalities in these patients. We sought to evaluate
imaging findings from patients with suspected or diagnosed FMD who underwent screening CTA at our
institution with a novel single-acquisition protocol that offers increased anatomic coverage, with images
obtained from the skull vertex to the pelvis.

Methods: Images from 80 consecutive patients scanned with the novel single-session CTA protocol covering the skull vertex to the pelvis were compared with images from 20 additional consecutive patients who underwent CTA for the head and neck separate from CTA of the chest, abdomen, and pelvis.

Results: Compared with CTA performed in separate sessions, the single-session CTA protocol decreased the radiation dose by $38 \%(\mathrm{P}<0.001)$ and decreased the contrast dose by $39 \%(\mathrm{P}<0.001)$, with satisfactory image quality noted in all instances. Additionally, higher mean contrast attenuation was noted in the aortic arch with use of the novel protocol $(409 \pm 76 \mathrm{HU})$ versus with use of the dual-acquisition protocol $(260 \pm 38$ $\mathrm{HU} ; \mathrm{P}<0.001)$.

Conclusions: These results suggest that use of a novel single-session CTA protocol extending from the skull vertex to the pelvis provides effective screening imaging in patients with suspected or diagnosed FMD as compared with multisession, standard-pitch CTA.

Keywords: Computed tomography angiography (CTA); fibromuscular dysplasia (FMD); screening

Submitted Nov 18, 2019. Accepted for publication Feb 07, 2020.

doi: 10.21037/cdt.2020.02.06

View this article at: http://dx.doi.org/10.21037/cdt.2020.02.06

\section{Introduction}

Fibromuscular dysplasia (FMD) is a nonatherosclerotic, noninflammatory, idiopathic, localized disease that can affect all layers of small and medium arteries (1) but most commonly affects medium-sized arteries, including renal, carotid, and vertebral arteries (2). Clinical manifestations of FMD are typically related to the vascular territory involved; for example, resistant hypertension may be seen in younger patients with renal artery stenosis, whereas carotid artery involvement may manifest as headache or pulsatile tinnitus and dissection of carotid or vertebral arteries may manifest as transient ischemic attack or stroke. Imaging is central to the diagnosis of FMD, with a recent classification of FMD based on angiographic findings, including multifocal (previously referred to as medial fibroplasia) and focal (previously referred to as intimal fibroplasia) subtypes (3).

Screening imaging of patients with suspected FMD requires a wide range of anatomic coverage while 


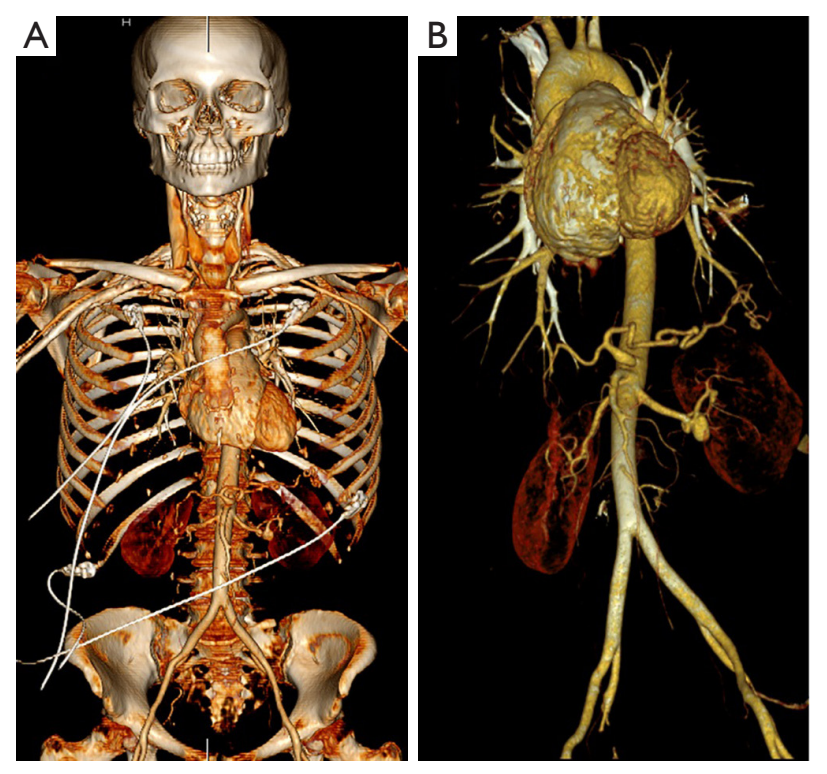

Figure 1 Images obtained from the skull vertex to the ischial tuberosities in a single session using the novel technique. Volumerendered images demonstrate the range of body coverage (A) and the thoracoabdominal aorta after bone removal (B).

maintaining adequate spatial resolution, acceptable ionizing radiation exposure and contrast burden, and reasonable time requirement. We previously reported the results of a study using a specialized screening computed tomography angiography (CTA) protocol to evaluate the chest, abdomen, and pelvis in patients enrolled in our institutional FMD registry (4). However, a more extensive range of screening imaging may be desirable, as noted in a recent international consensus document; this document advises that all patients with FMD, regardless of the initial site of involvement, should undergo CTA or contrast-enhanced magnetic resonance angiography of all vessels from the brain to the pelvis so that clinicians can identify other areas of FMD and screen for occult aneurysms and dissections (5). Ideally, this imaging would be completed in one visit. At our institution, we have instituted a novel screening CTA protocol for patients with suspected FMD that allows for the assessment of vascular territories extending from the skull vertex to the pelvis as part of one contemporaneously acquired data set. For this study, we reviewed the cases of patients imaged with this specialized protocol and assessed the image quality, radiation doses, and contrast doses in these patients versus in patients who underwent CTA scans covering a similar anatomic range but completed in two separate sessions.

\section{Methods}

This retrospective study was approved by the local institutional review board with a waiver of informed consent. For this study, we searched an institutional imaging database to identify cases of patients who underwent CTA for suspected FMD. Group 1 consisted of 80 consecutive patients who, between January 2018 and March 2019, underwent a novel high-pitch CTA protocol with images extending from the skull vertex to the pelvis. Group 2 consisted of 20 consecutive patients who, between August 2015 and February 2019, underwent standard-pitch CTA of the head and neck separate from high-pitch CTA of the chest, abdomen, and pelvis (mean interval between scans, $264 \pm 247$ days). No patients were excluded from either group.

\section{CT image acquisition and analysis}

For patients in group 1, we used a novel high-pitch CTA protocol to obtain images from the skull vertex to the ischial tuberosities (Figure 1) in a single session. CT scans were obtained on a Siemens (Erlangen, Germany) dual-source scanner (Definition Force $2 \times 192$ slice or Definition Flash $2 \times 128$ slice). Electrocardiogram (ECG) triggering and a fixed technique $(350 \mathrm{mAs}$ and $120 \mathrm{kV}$ ) were used. Patients kept their arms at their sides during scanning to allow for adequate assessment of the neck and skull base. The neck and skull base images were reconstructed at $0.75 \mathrm{~mm}$ slice thickness, whereas the chest, abdomen, and pelvis images were reconstructed at both 3 and $1 \mathrm{~mm}$ slice thickness. A weight-based dosing regimen of intravenous contrast was used, with Omnipaque 350 administered to 78 patients and Omnipaque 300 administered to 2 patients.

Patients in group 2 underwent high-pitch, ECG-triggered CTA of the chest, abdomen, and pelvis as previously described (4) and standard-pitch CTA of the head and neck. Scanner $\mathrm{kV}$ and $\mathrm{mAs}$ settings were determined by patient body habitus and internally developed software. Omnipaque 350 was administered to all patients.

Images were analyzed at dedicated workstations by two radiologists with cardiovascular imaging fellowship training (7 and 12 years of postresidency experience). Agfa IMPAX 6 (Mortsel, Belgium) software was used for image analysis. Images were reviewed for the presence of convincing beading, focal stenosis, diffuse ectasia, aneurysm, and dissection. Axial images were initially reviewed, with additional observations made using multiplanar reformatted images, as well as maximum intensity projection images in 
Table 1 Patient demographics

\begin{tabular}{lccc}
\hline Characteristic & Group 1 & Group 2 & P value \\
\hline Age, mean \pm SD (years) & $53 \pm 12$ & $55 \pm 14$ & 0.47 \\
Height, mean \pm SD $(\mathrm{cm})$ & $166 \pm 8$ & $156 \pm 33$ & 0.02 \\
Weight, mean \pm SD $(\mathrm{kg})$ & $75 \pm 18$ & $75 \pm 20$ & 0.99 \\
Body mass index, & $27 \pm 6$ & $29 \pm 11$ & 0.24 \\
mean \pm SD $\left(\mathrm{kg} / \mathrm{m}^{2}\right)$ & & & \\
Sex $(\%)$ & & & \\
Male & 11 & 10 & \\
Female & 89 & 90 & \\
\hline
\end{tabular}

multiple planes. For both groups, contrast enhancement was evaluated by measuring mean attenuation (in Hounsfield units, HU) at the level of the aortic arch (using a $2-\mathrm{cm}$ circle) and from the abdominal aorta at the level of the renal arteries (using a $1-\mathrm{cm}$ circle). We chose to measure aortic attenuation in these areas as a surrogate for measuring satisfactory enhancement within the branch vessels arising at these two levels, as these vessels are relatively small and can be difficult to assess with manually drawn regions of interest.

Radiation exposure was estimated with dose length product (DLP) values generated by the scanner at the time of the scan. Image quality was graded visually as high, medium, or low at levels of the aortic root and the small and medium arteries, based on image suitability for anatomic delineation and depiction of pathologic changes. As noted by other investigators, the final image quality is dependent on both patient and technical factors and represents a combined entity, with no single objective scale available (6). In this investigation, as is often the case in clinical practice, the factors that influenced CTA quality were those that affected image interpretation, most commonly image noise, vascular enhancement, and motion within the acceptable range (7). Categories of image quality were therefore defined as follows: high quality: clear anatomic and pathologic depiction with no imaging limitations; medium quality: anatomic and pathologic delineation slightly degraded but still judged as satisfactory for diagnostic purposes; and low quality: compromised anatomic and pathologic assessment, possibly limiting diagnostic yield of study.

\section{Statistical analysis}

Data are presented as mean values $\pm \mathrm{SD}$. $\mathrm{P}$ values were calculated for comparisons between groups. Statistical analysis was performed using Excel (Microsoft, Redmond, WA, USA).

\section{Results}

Patients in groups 1 and 2 demonstrated no significant differences in age, weight, or body mass index (Table 1).

Use of a specialized CTA protocol in a single session (group 1) decreased the estimated radiation dose by $38 \%$ versus use of a dual-acquisition protocol in separate sessions (group 2) (group 1: mean DLP, $579 \pm 63 \mathrm{mGy}^{*} \mathrm{~cm}$; group 2: mean combined DLP, $\left.929 \pm 296 \mathrm{mGy}^{*} \mathrm{~cm} ; \mathrm{P}<0.001\right)$. Image quality was preserved, with all patients in both groups having satisfactory or greater ratings in all cases [group 1: high quality, $60 \%$ [48/80]; medium quality, 40\% (32/80); group 2: high quality, $65 \%$ (13/20); medium quality, 35\% (7/20)]. Patients in group 1 also demonstrated a reduction in contrast dose of $39 \%$ (group 1: mean amount of contrast, $120 \pm 27 \mathrm{~mL}$; group 2: mean combined amount of contrast, $198 \pm 12 \mathrm{~mL} ; \mathrm{P}<0.001$ ).

Contrast attenuation in the arch was excellent in group $1(409 \pm 76 \mathrm{HU})$, but enhancement in group 2 was on the borderline of satisfactory $(260 \pm 38 \mathrm{HU})(\mathrm{P}<0.001) ; 250$ $\mathrm{HU}$ has often been cited as representing satisfactory aortic luminal enhancement (8). Although group 2 showed statistically higher contrast at the abdominal level, this result was not clinically relevant, as both groups showed excellent enhancement at this level (group 1, 402 $\pm 68 \mathrm{HU}$; group 2, $479 \pm 158 \mathrm{HU} ; \mathrm{P}=0.001$ ).

We observed evidence of arterial beading (Figure 2), focal stenosis, dissection (Figure 3), diffuse ectasia (Figure 4), and aneurysms in the extracranial internal carotid arteries and in the vertebral, renal, iliac, celiac trunk, mesenteric, splenic, and hepatic distributions. The distribution of vessel involvement is listed for each group in Table 2 and for the combined groups in Table 3. Figures 5,6 represent images graded as high quality and medium quality, respectively.

\section{Discussion}

In this study, we found that in patients with suspected FMD, a novel single-session high-pitch CTA protocol extending from the skull vertex to the pelvis was associated with lower radiation and contrast doses when compared with standardpitch CTA for the head and neck and separate CTA of the chest, abdomen, and pelvis, with satisfactory image quality in all instances. Additionally, improved contrast attenuation was noted at the aortic arch level in patients undergoing the novel CTA protocol, with both groups showing excellent contrast enhancement at the level of the renal arteries.

The CTA protocol in this investigation allows for wide anatomic range coverage to be obtained in a single visit. Previously, using standard-pitch imaging and following the 

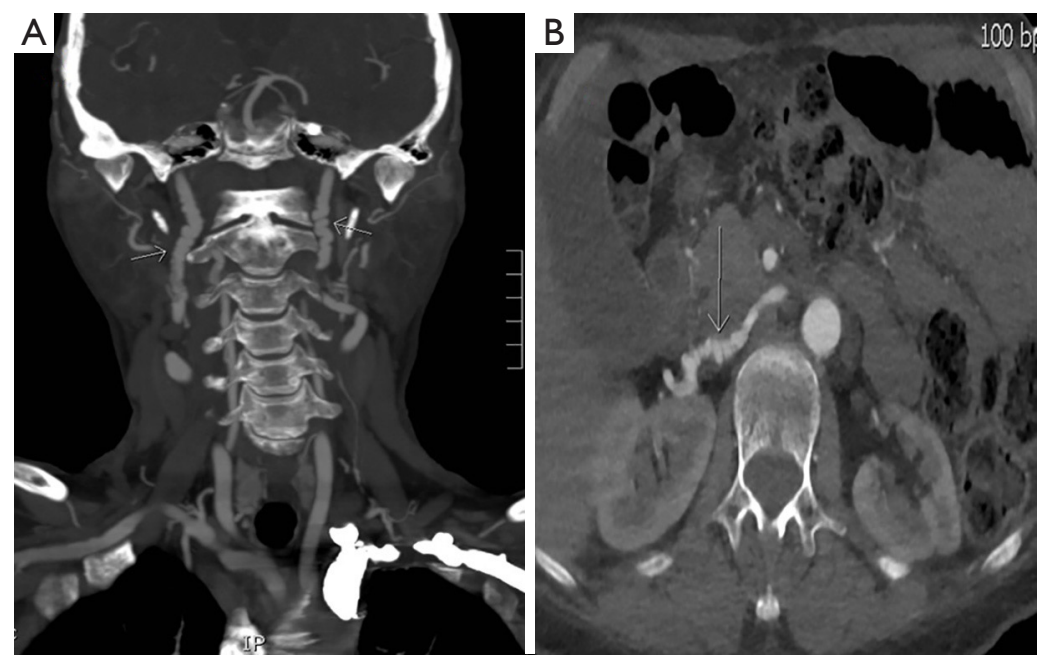

Figure 2 Images of arterial beading obtained using the novel technique. Fibromuscular dysplasia (arrows) demonstrated on coronal multiplanar images involving the bilateral carotid arteries (A) and axial images of the right renal artery (B) in 2 different patients.
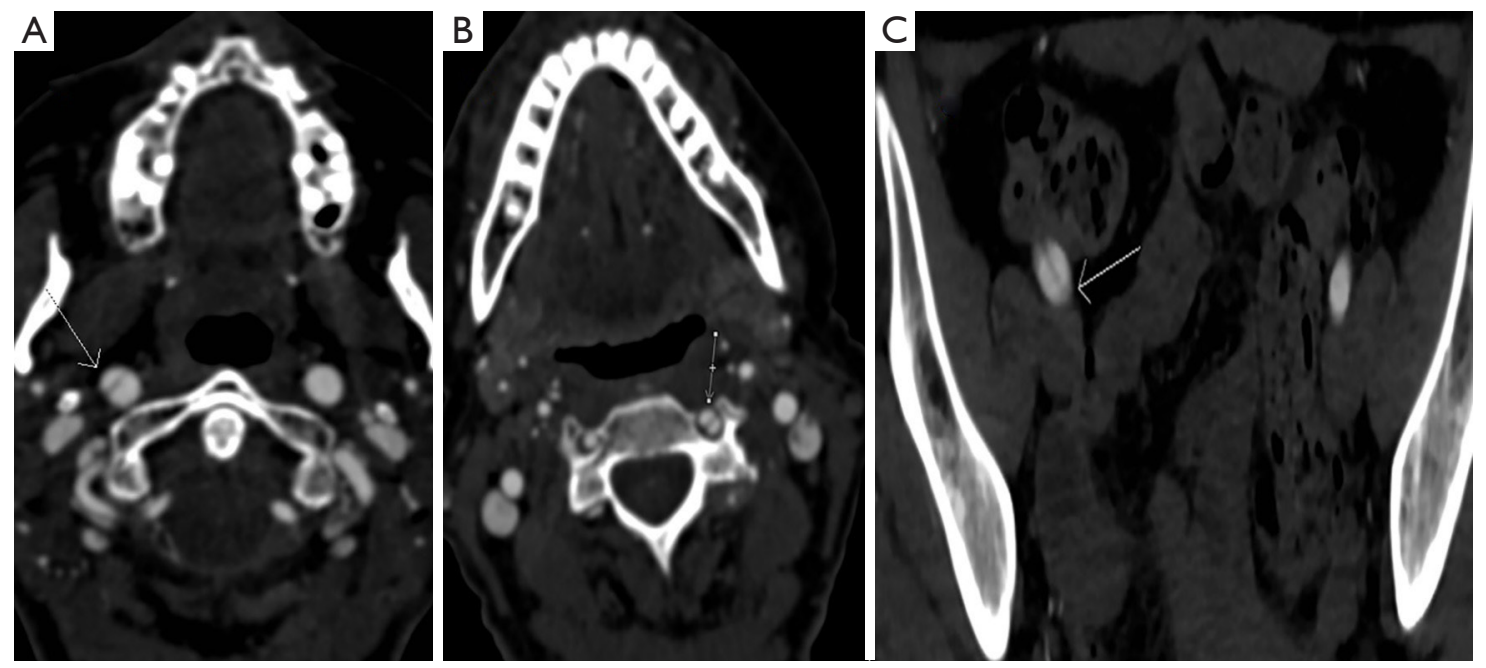

Figure 3 Dissections (arrows) in 3 different patients with fibromuscular dysplasia. Axial images obtained using the novel technique demonstrate dissection of the right common carotid artery (A), left vertebral artery (B), and right external iliac artery (C).

contrast administration policy used by our institution (jointly established by the radiology and nephrology departments) required that CTA images extending from the skull vertex to the pelvis be obtained in a single session with a suboptimal contrast agent or in two separate imaging encounters. Use of the novel CTA protocol described in this study allows us to follow the "as low as reasonably achievable" (ALARA) imaging mandate, providing diagnostic quality imaging with minimization of radiation burden $(9,10)$.

In our study, the most commonly affected arteries were the extracranial internal carotid arteries (49\%) followed by the left $(38 \%)$ and right $(31 \%)$ vertebral arteries and the right (20\%) and left (16\%) renal arteries. A previous study of 447 cases from the FMD registries of nine sites found that the most commonly affected arteries were the renal arteries (79.7\%), the extracranial carotid $(74.4 \%)$ and vertebral $(36.6 \%)$ arteries, the mesenteric arteries $(26 \%)$, and the intracranial carotid arteries (17\%) (11). The reason for these observed differences in vessel territory involvement is not entirely clear but is likely at least in part attributable to 

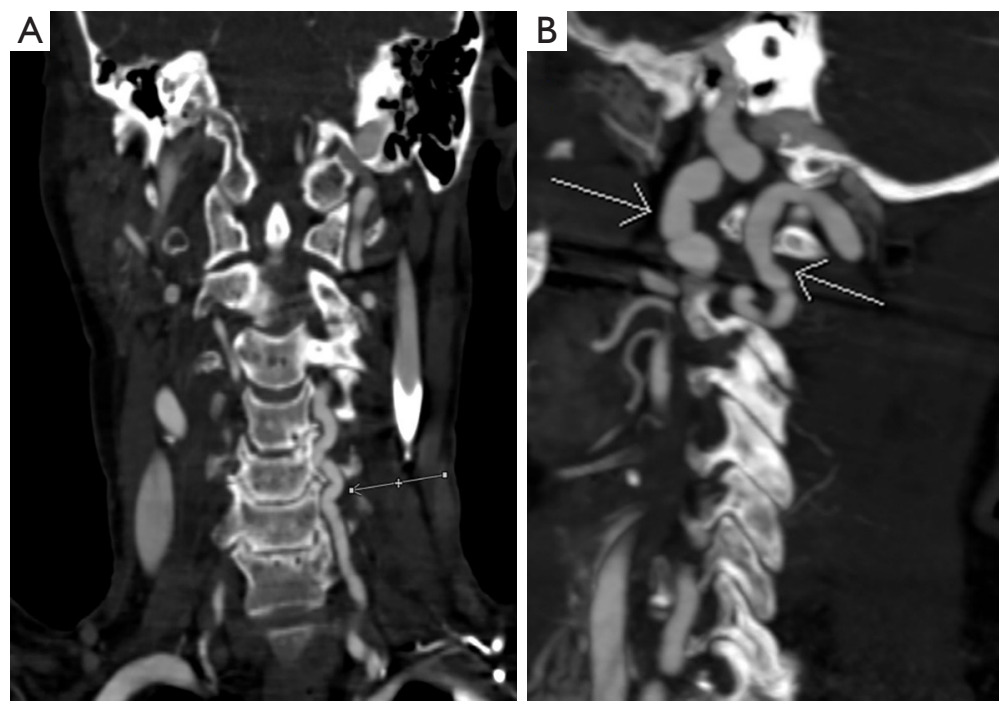

Figure 4 Multiplanar reformatted images obtained using the novel imaging technique. (A) Diffuse ectasia and tortuosity of the vessel (arrows) demonstrated on a coronal reformatted image of the left vertebral artery; (B) ectasia and tortuosity of the left common carotid and left vertebral arteries (arrows) demonstrated on a sagittal maximum intensity projection image.

Table 2 Distribution of vessel involvement for each group

\begin{tabular}{lcc}
\hline Vessel involvement & Group 1, n [\%] & Group 2, n [\%] \\
\hline Right renal & $16[20]$ & $4[20]$ \\
Left renal & $14[18]$ & $2[10]$ \\
Right common iliac & $2[3]$ & $1[5]$ \\
Left common iliac & $4[5]$ & 0 \\
Right external iliac & $8[10]$ & $2[10]$ \\
Left external iliac & $6[8]$ & $2[10]$ \\
Right internal iliac & $2[3]$ & 0 \\
Left internal iliac & $3[4]$ & 0 \\
Other abdominal visceral & $15[19]$ & $4[20]$ \\
Right common carotid & 0 & 0 \\
Left common carotid & 0 & 0 \\
Right internal carotid & $34[43]$ & $15[75]$ \\
Left internal carotid & $36[45]$ & $13[65]$ \\
Right vertebral & $20[25]$ & $11[55]$ \\
Left vertebral & $25[31]$ & $13[65]$ \\
Anterior circulation of Willis & $5[6]$ & $1[5]$ \\
Posterior circulation of Willis & $3[4]$ & $1[5]$ \\
\hline & & \\
\hline
\end{tabular}

Table 3 Distribution of vessel involvement for both groups

\begin{tabular}{|c|c|}
\hline Vessel involvement & Groups 1 and 2, n [\%] \\
\hline Right renal & $20[20]$ \\
\hline Left renal & $16[16]$ \\
\hline Right common iliac & $3[3]$ \\
\hline Left common iliac & $4[4]$ \\
\hline Right external iliac & 10 [10] \\
\hline Left external iliac & 8 [8] \\
\hline Right internal iliac & 2 [2] \\
\hline Left internal iliac & $3[3]$ \\
\hline Other visceral ${ }^{\star}$ & 19 [19] \\
\hline Celiac trunk & 11 \\
\hline Splenic artery & 9 \\
\hline Superior mesenteric artery & 6 \\
\hline Hepatic artery & 1 \\
\hline Right common carotid & 0 \\
\hline Left common carotid & 0 \\
\hline Right internal carotid & 49 [49] \\
\hline Left internal carotid & 49 [49] \\
\hline Right vertebral & $31[31]$ \\
\hline Left vertebral & $38[38]$ \\
\hline Anterior circulation of Willis & $6[6]$ \\
\hline Posterior circulation of Willis & $4[4]$ \\
\hline
\end{tabular}

*, some of these cases involved abnormalities in multiple vessels. 

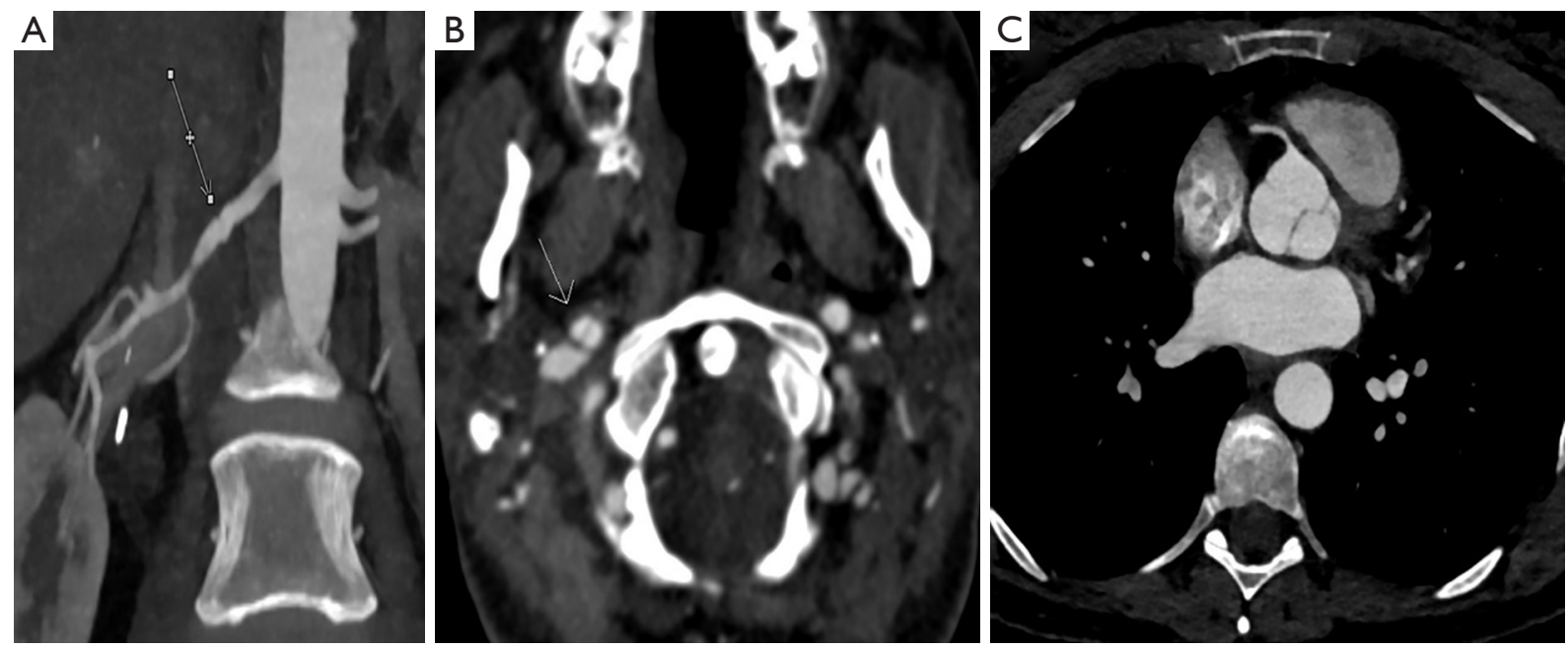

Figure 5 High-quality images obtained using the novel technique. Right renal beading demonstrated on a coronal maximum intensity projection image (A); right carotid dissection/pseudoaneurysm (arrow) demonstrated on an axial image (B); on an axial image, the level of the aortic root is demonstrated $(\mathrm{C})$.
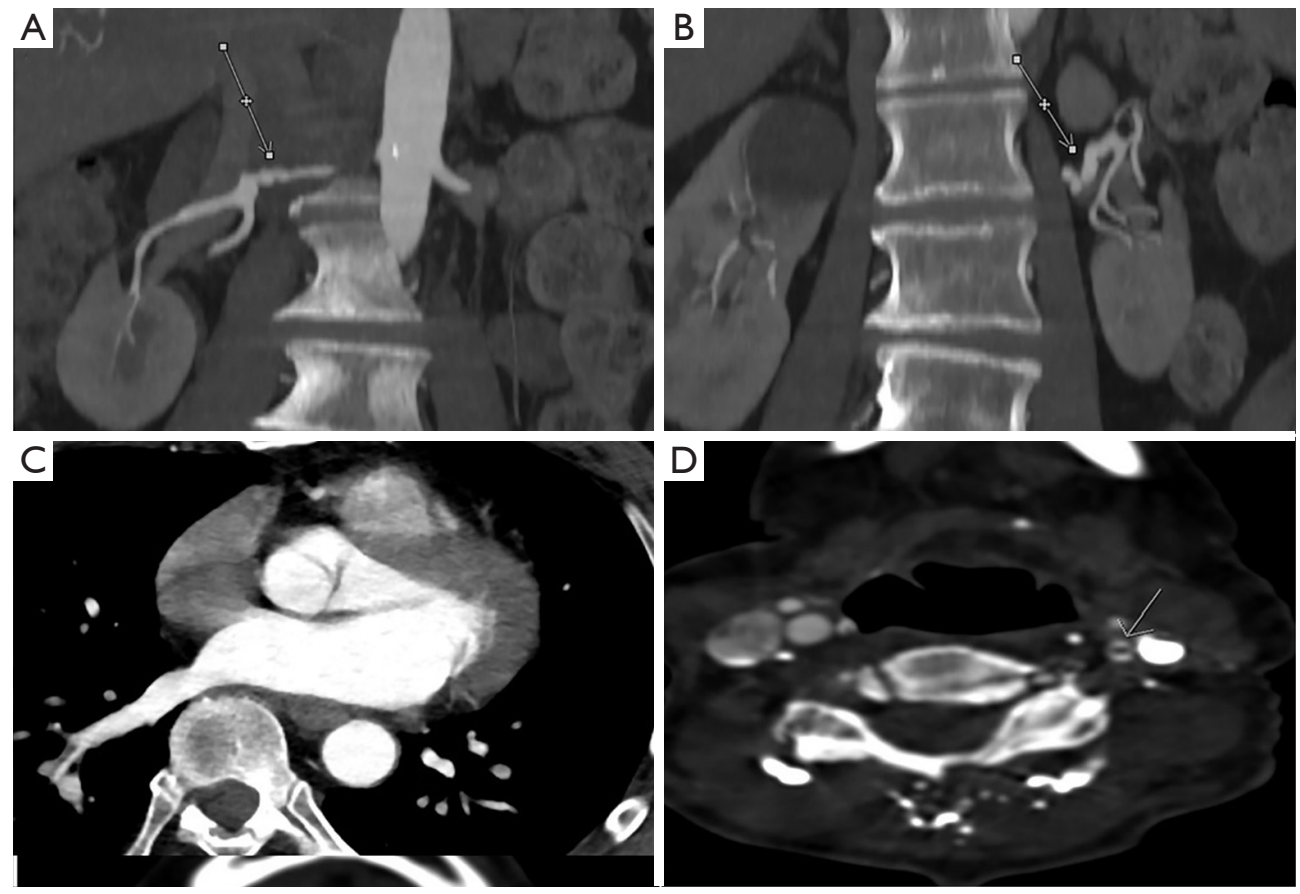

Figure 6 Medium-quality images obtained using the novel technique. Right renal (A) and left renal (B) beading demonstrated on coronal maximum intensity projection images. On axial images, the level of the aortic root (C) and a left carotid dissection (D) from different patients can be seen.

heterogeneity among patients referred for imaging studies.

This study was limited by its retrospective nature. Additionally, study patients were scanned at a single center.
The CTA technique we used requires access to highpitch imaging scanner technology, which is not universally available. We must also note the heterogeneity of the two 
different patient groups involved in this study. Finally, we acknowledge the limitation of using a subjective grading system to evaluate image quality in this investigation, as well as the lack of a "gold standard" for the diagnosis of FMD that would have allowed us to analyze diagnostic accuracy. However, the physicians who interpreted the images in this investigation have subspecialty training in cardiovascular radiology and are exposed to a high volume of dedicated imaging studies for suspected FMD. The clinical standard of diagnosis for FMD is dependent, at least in part, on a similar subjective assessment of arterial imaging findings; nonetheless, we wanted to provide a comparator for our novel protocol.

\section{Conclusions}

In conclusion, use of a novel high-pitch CTA protocol extending from the skull vertex to the pelvis allows for effective FMD screening in a single visit, with preserved image quality, improved contrast attenuation, and decreased contrast and radiation burden when compared with a dualacquisition CTA protocol. Further validation of this approach could be obtained with larger, multicenter investigations.

\section{Acknowledgments}

The authors would like to acknowledge Megan Griffiths for her assistance in manuscript preparation and acknowledge Erika Schneider for her valuable assistance in data collection. Funding: None.

\section{Footnote}

Conflicts of Interest: Both authors have completed the ICMJE uniform disclosure form (available at http://dx.doi. org/10.21037/cdt.2020.02.06). The authors have no conflicts of interest to declare.

Ethical Statement: The authors are accountable for all aspects of the work in ensuring that questions related to the accuracy or integrity of any part of the work are appropriately investigated and resolved. This retrospective study was approved by the local institutional review board with a waiver of informed consent.

Open Access Statement: This is an Open Access article distributed in accordance with the Creative Commons Attribution-NonCommercial-NoDerivs 4.0 International License (CC BY-NC-ND 4.0), which permits the non- commercial replication and distribution of the article with the strict proviso that no changes or edits are made and the original work is properly cited (including links to both the formal publication through the relevant DOI and the license). See: https://creativecommons.org/licenses/by-nc-nd/4.0/.

\section{References}

1. Plouin PF, Perdu J, La Batide-Alanore A, et al. Fibromuscular dysplasia. Orphanet J Rare Dis 2007;2:28.

2. Touzé E, Oppenheim C, Trystram D, et al. Fibromuscular dysplasia of cervical and intracranial arteries. Int J Stroke 2010;5:296-305.

3. Olin JW, Gornik HL, Bacharach JM, et al. Fibromuscular dysplasia: state of the science and critical unanswered questions: a scientific statement from the American Heart Association. Circulation 2014;129:1048-78.

4. Bolen MA, Brinza E, Renapurkar RD, et al. Screening CT angiography of the aorta, visceral branch vessels, and pelvic arteries in fibromuscular dysplasia. JACC Cardiovasc Imaging 2017;10:554-61.

5. Gornik HL, Persu A, Adlam D, et al. First International Consensus on the diagnosis and management of fibromuscular dysplasia. Vasc Med 2019;24:164-89.

6. Ghekiere O, Nchimi A, Djekic J, et al. Coronary Computed Tomography Angiography: Patient-related factors determining image quality using a second-generation 320-slice CT scanner. Int J Cardiol 2016;221:970-6.

7. Ghekiere O, Salgado R, Buls N, et al. Image quality in coronary CT angiography: Challenges and technical solutions. Br J Radiol 2017;90:20160567.

8. Bae KT. Optimization of contrast enhancement in thoracic MDCT. Radiol Clin North Am 2010;48:9-29.

9. McCollough CH, Primak AN, Braun N, et al. Strategies for reducing radiation dose in CT. Radiol Clin North Am 2009;47:27-40.

10. Yu L, Liu X, Leng S, et al. Radiation dose reduction in computed tomography: techniques and future perspective. Imaging Med 2009;1:65-84.

11. Olin JW, Froehlich J, Gu X, et al. The United States Registry for Fibromuscular Dysplasia: results in the first 447 patients. Circulation 2012;125:3182-90.

Cite this article as: Abozeed M, Bolen MA. Screening CT angiography in patients with suspected fibromuscular dysplasia: improved patient care with single-session skull vertex to pelvis coverage. Cardiovasc Diagn Ther 2020;10(2):201-207. doi: 10.21037/cdt.2020.02.06 